\title{
Low Concentrations of Dehydroepiandrosterone Sulfate are Associated with Depression and Fatigue in Patients with Non-Small-Cell Lung Cancer After Chemotherapy
}

This article was published in the following Dove Press journal:

Neuropsychiatric Disease and Treatment

Yu-Hung Fang, (iD) 'Meng-

Jer Hsieh, (iD) 2,3 Ming-Szu Hung, 1,4,5

Yu-Ching Lin, (D) ${ }^{1,3-5}$ Liang-

Tseng Kuo, ${ }^{5,6}$ Mong-Liang Lu, ${ }^{7}$

Bor-Show Tzang, ${ }^{8-10}$

Vincent Chin-Hung Chen ${ }^{5,11}$

'Division of Thoracic Oncology, Department of Pulmonary and Critical Care Medicine,

Chang Gung Memorial Hospital, Chiayi,

Taiwan; ${ }^{2}$ Department of Respiratory Therapy,

College of Medicine, Chang Gung University,

Chiayi, Taiwan; ${ }^{3}$ Division of Pulmonary

Infection and Critical Care Medicine,

Department of Pulmonary and Critical Care

Medicine, Chang Gung Memorial Hospital,

Chiayi, Taiwan; ${ }^{4}$ Department of Respiratory

Care, Chang Gung University of Science and

Technology, Chiayi, Taiwan; ${ }^{5}$ Department of

Medicine, College of Medicine, Chang Gung

University, Taoyuan, Taiwan; ${ }^{6}$ Division of

Sports Medicine, Department of Orthopaedic

Surgery, Chang Gung Memorial Hospital,

Chiayi, Taiwan; ${ }^{7}$ Department of Psychiatry,

Wan Fang Hospital, and School of Medicine,

College of Medicine, Taipei Medical University,

Taipei, Taiwan; ${ }^{8}$ Institute of Biochemistry,

Microbiology and Immunology, Chung Shan

Medical University, Taichung, Taiwan; ${ }^{9}$ Clinical

Laboratory, Chung Shan Medical University

Hospital, Taichung, Taiwan; ${ }^{10}$ Department of

Biochemistry, School of Medicine, Chung Shan

Medical University, Taichung, Taiwan;

"Department of Psychiatry, Chang Gung

Memorial Hospital, Chiayi, Taiwan

Correspondence: Vincent Chin-Hung Chen Department of Psychiatry, Chang Gung

Memorial Hospital, Chiayi Branch, No. 6,

W. Sec., Jiapu Road, Puzi City, Chiayi County

61363, Taiwan, Republic of China

Tel +886-5-3621000 ext. 2315

Email cch1966@gmail.com
Background: Dehydroepiandrosterone (DHEA) and DHEA sulfate (DHEAS) concentrations were reported to decrease in patients with advanced cancer. However, the clinical significance of DHEA and DHEAS concentrations in patients with NSCLC receiving chemotherapy (CT) has not been sufficiently documented.

Objective: To evaluate the correlation between mental health and hormone concentrations on patients with advanced non-small-cell lung cancer (NSCLC).

Materials and Methods: The present study was a cross-sectional analysis based on a selfreported psychological investigation. Salivary samples were collected from 22 patients with advanced NSCLC after CT and 17 healthy controls. The concentrations of DHEA, DHEAS, and cortisol were analyzed to investigate their associations with the results of self-reported questionnaires on psychological health.

Results: Patients with advanced NSCLC exhibited significantly higher Patient Health Questionnaire (PHQ-9) and Startle, Physiological arousal, Anger, and Numbness-Chinese version (SPAN-C) scores, poorer health conditions, lower sleep quality, and more severe fatigue after CT than did healthy controls, and salivary concentrations of DHEA and DHEAS were significantly lower among patients after CT than among controls. DHEAS concentrations were negatively associated with depression scores (PHQ-9, $\mathrm{r}=-0.496, P=0.019$ ) and fatigue scores (Brief Fatigue Inventory-Taiwan, $\mathrm{r}=-0.562, P=0.006$ ).

Conclusion: Patients with advanced NSCLC after CT had lower DHEA and DHEAS concentrations than did controls. Lower DHEAS concentrations were associated with higher fatigue and depression scores.

Keywords: cancer-related fatigue, chemotherapy, DHEA, DHEAS, lung cancer

\section{Background}

Cancer is among the leading causes of death worldwide. Lung cancer is the most commonly diagnosed cancer and the leading cause of cancer-related deaths worldwide. ${ }^{1}$ However, the overall survival rate among patients with non-small-cell lung cancer (NSCLC) has improved in the past two decades. ${ }^{2}$ With the prolonged survival time of lung cancer patients, psychosocial health is the same important in lung cancer patients. Compared with other cancers, lung cancer remains insufficiently studied from the viewpoint of psychosocial health. ${ }^{3}$ Despite the advantages of targeted therapy and immunotherapy, platinum-based chemotherapy (CT) remains the gold 
standard for most patients with lung cancer. ${ }^{4}$ Over the course of $\mathrm{CT}$, most patients with lung cancer experience pain, depression, and fatigue. ${ }^{5}$ Previous studies have reported that patients with lung cancer exhibited the highest intensity of stress among patients with 14 types of cancer, ${ }^{6}$ and these patients also exhibited the highest prevalence of major depression disorder among patients with 10 types of cancer. $^{7}$ On average, depression affects $23-40 \%$ of patients with lung cancer, and approximately $43 \%$ of patients with lung cancer experienced moderate to severe fatigue. ${ }^{89}$

Current theories regarding mechanisms underlying cancer-related fatigue (CRF) and depression were both associated with hypothalamic-pituitary-adrenal (HPA) axis dysfunction. ${ }^{10,11}$ Dehydroepiandrosterone (DHEA) and DHEA sulfate (DHEAS) are androgen precursors synthesized and secreted primarily by the adrenal cortex. Compared with DHEA, DHEAS has a longer half-life and fewer circadian variations. ${ }^{12}$ Low serum concentrations of DHEA and DHEAS are associated with metabolic and cardiovascular diseases, decline in muscle mass, poor physical performance, mood changes, and memory deficits. ${ }^{13}$ Patients with lung cancer were reported to have lower concentrations of serum DHEAS, ${ }^{14}$ and patients with advanced cancer were reported to exhibit low endogenous DHEAS production. ${ }^{15}$ DHEA and DHEAS concentrations were both substantially lower during targeted therapy with gefitinib than pretreatment values in patients with lung cancer. ${ }^{16}$ However, the effects of DHEA or DHEAS concentrations on patients with lung cancer receiving CT have not been satisfactorily established thus far. We do not yet know the exact physiologic or psychological effect of stress-related hormone in cancer patients. Limited research has been conducted on the neuroendocrine system in cancer patients with cancer-related fatigue and depression. In the present study, the association between hormones and psychological health in patients with lung cancer receiving CT was evaluated.

\section{Materials and Methods}

\section{Participants}

The present study is a prospective, cross-sectional analysis based on a self-reported psychological investigation. We included patients at least 20 years with pathologically proven primary NSCLC who received CT at Chiayi Chang Gang Memorial Hospital between November 2017 and June 2019. Patients were included if they had stage IIIb or IV NSCLC. Platinum-based chemotherapy regimen used in our patients could be cisplatin or carboplatin plus a third-generation agent (ie, gemcitabine, vinorelbine, a taxane, or pemetrexed) according to National Comprehensive Cancer Network (NCCN) guideline of NSCLC.${ }^{17}$ The post-CT period was defined as 3-6 months after platinum-based CT treatment. Demographically matched healthy participants with no recent infections or cancer history at the time of the evaluation were recruited through an advertisement at the hospital. In total, 66 patients met the eligibility requirements, but 44 refused to participate. Finally, we enrolled 22 NSCLC patients in the post-CT group and 17 healthy participants in the control group.

Clinical staging was determined according to the Cancer Staging Manual of the American Joint Committee on Cancer, 7 th edition, ${ }^{18}$ and the staging was confirmed by multidisciplinary committees. Patients were excluded if they had an Eastern Cooperative Oncology Group (ECOG) performance status ${ }^{19}$ of $>2$, were unable to complete questionnaires, did not provide informed consent, had brain metastases, or received brain radiotherapy or surgical interventions.

The Institutional Review Board of Chang Gung Memorial Hospital approved the protocol (201700297B0C604) followed in the present study and conformed to the Helsinki Declaration. Each participant was assigned an ID code for confidentiality and anonymity. All data were collected and classified using these ID codes. Anonymized data were recorded, and analyses were conducted using SPSS (SPSS Inc., Chicago, Ill., USA). Confidentiality of participants was respected and preserved throughout the trial. All investigations were performed after written informed consent was provided by participants.

\section{Questionnaires}

We used previously validated questionnaires such as depression (Patient Health Questionnaire [PHQ-9]), ${ }^{20}$ family support (Family APGAR index), ${ }^{21}$ post-traumatic stress disorder in terms of Startle, Physiological arousal, Anger, and Numbness-Chinese version (SPAN-C), ${ }^{22,23}$ general health status (EQ-5D visual analog scale [EQ-5D VAS]), ${ }^{24}$ fatigue (Brief Fatigue Inventory-Taiwan), ${ }^{25}$ sleep quality (Pittsburgh Sleep Quality Index, [PSQI]), ${ }^{26}$ and anxiety (Hospital Anxiety and Depression Scale, [HADS]), for investigating the health status of participants. ${ }^{27}$ Lung cancer patients receiving chemotherapy regular follow up at our pulmonary and oncology 
outpatient clinics were invited consecutively to take part in this study. Once our research assistants received referral from in-charge doctors or case manager, they went to the above settings to contact patients. After explaining the study procedure and aims, those who agreed to sign an informed consent form were enrolled in the study. Research assistants under the supervision of a psychiatrist and clinical psychologists administered these questionnaires.

\section{Salivary Stress Hormone Assay}

Before saliva collection, we first checked the oral health of participants. To avoid the interference of blood stress hormone, participants who exhibited oral conditions with bleeding, such as periodontal disease or wounds in the mouth, were excluded. Participants were asked to rinse their mouths thoroughly with cold water. After $10 \mathrm{~min}$, $2 \mathrm{~mL}$ of saliva was collected in a polypropylene tube during the daytime. Colorless saliva samples were transported to the laboratory in ice bags. The saliva samples were centrifuged at $2000 \times \mathrm{g}$ for $10 \mathrm{~min}$ at $4^{\circ} \mathrm{C}$. Aliquots of the supernatant were stored at $-80^{\circ} \mathrm{C}$. The salivary DHEA, DHEAS, and cortisol concentrations were evaluated using the enzyme-linked immunosorbent assay, following the manufacturer's instruction manual (IBL International, Germany, RE62051, RE52661, and RE52611). Both a standard curve and interpolation were applied to convert the absorbance value into the hormone concentration.

\section{Statistical Analysis}

Differences between groups were analyzed using Pearson's chi-square test, and analysis of variance was used to examine categorical variables. The independent sample Kruskal-Wallis test was used for all continuous variables because of the small sample size and unequal distribution among groups. We used Spearman's rank correlation to evaluate the correlation between neuroendocrine hormone concentrations and psychological health data. The threshold for statistical significance was set at 5\%. All analyses were performed using PASW Statistics 18 (SPSS Inc., USA).

\section{Results}

The two groups did not differ statistically in age, sex, educational level, or demographic status (Table 1). The percentages of visits to psychiatrists and psychiatric medication prescriptions tended to be higher in the post-CT
Table I Sociodemographic and Clinical Data of Patients with Lung Cancer

\begin{tabular}{|c|c|c|c|}
\hline Variables & $\begin{array}{l}\text { Post- } \\
\text { Chemotherapy } \\
(\mathrm{N}=22)\end{array}$ & $\begin{array}{l}\text { Healthy } \\
\text { Control } \\
(\mathbf{N}=17)\end{array}$ & $P$ \\
\hline & $\mathbf{N}(\%)$ & $\mathbf{N}(\%)$ & \\
\hline Age, mean $\pm S D$ & $62.09 \pm 5.79$ & $62.35 \pm 8.99$ & 0.913 \\
\hline $\begin{array}{l}\text { Education (years), mean } \\
\pm \mathrm{SD}\end{array}$ & $9.82 \pm 4.24$ & $10.65 \pm 4.54$ & 0.561 \\
\hline $\begin{array}{l}\text { Sex } \\
\qquad \text { Female } \\
\text { Male }\end{array}$ & $\begin{array}{l}16(72.7 \%) \\
6(27.3 \%)\end{array}$ & $\begin{array}{l}10(58.8 \%) \\
7(41.2 \%)\end{array}$ & 0.361 \\
\hline $\begin{array}{l}\text { Marriage } \\
\text { Unmarried } \\
\text { Married }\end{array}$ & $\begin{array}{l}\text { I ( } 4.5 \%) \\
2 \text { I (95.5\%) }\end{array}$ & $\begin{array}{l}0 \\
17(100 \%)\end{array}$ & 0.373 \\
\hline $\begin{array}{l}\text { Living Status } \\
\text { Live alone } \\
\text { Live with others }\end{array}$ & $\begin{array}{l}2(9.1 \%) \\
20(90.9 \%)\end{array}$ & $\begin{array}{l}0 \\
17(100 \%)\end{array}$ & 0.202 \\
\hline $\begin{array}{l}\text { Smoking } \\
\text { Current } \\
\text { Former } \\
\text { Never }\end{array}$ & $\begin{array}{l}\text { I (4.5\%) } \\
\text { I } 3(59.1) \\
8(36.4 \%)\end{array}$ & $\begin{array}{l}0 \\
5(29.4 \%) \\
12(70.4 \%)\end{array}$ & 0.091 \\
\hline Psychiatrist visit & $5(22.7 \%)$ & I (5.9\%) & 0.361 \\
\hline Psychiatric medication & $6(27.3 \%)$ & I (5.9\%) & 0.222 \\
\hline
\end{tabular}

Abbreviation: SD, standard deviation.

group than in the control group; however, the trends were not significantly different (Table 1).

The post-CT group exhibited significantly higher PHQ9 scores and lower EQ-5D VAS scores than did the control group $(6.45 \pm 6.05$ vs $1.00 \pm 1.80, P<0.001 ; 59.55 \pm$ 19.45 vs $75.29 \pm 14.19, P=0.008$, respectively, Table 2 ). The post-CT group also exhibited lower sleep quality, which was measured using PSQI scores, than did the control group $(8.31 \pm 5.88$ vs $4.43 \pm 2.53, P=0.002$, Table 2). The salivary concentrations of DHEA and DHEAS were significantly lower in the post-CT group than in the control group $(75.00 \pm 63.31$ vs $167.56 \pm$ 127.16, $P=0.012 ; 2.12 \pm 1.32$ vs $3.29 \pm 1.96, P=$ 0.032 , respectively, Table 2 ).

To evaluate the correlation between salivary stress hormones and psychological health data, we used Spearman's rank correlation. However, the salivary concentrations of DHEA, DHEAS, and cortisol were neither associated with family support nor with SPAN-C, EQ-5D VAS, PSQI, and HADS scores. We observed that fatigue 
Table 2 Psychological Health and Salivary Hormone Data of Patients with Lung Cancer

\begin{tabular}{|l|l|l|l|}
\hline & $\begin{array}{l}\text { Post- } \\
\text { Chemotherapy } \\
\text { (N = 22) }\end{array}$ & $\begin{array}{l}\text { Healthy } \\
\text { Control } \\
\text { (N =17) }\end{array}$ & $\mathbf{P}$ \\
\hline $\begin{array}{l}\text { Psychological health } \\
\text { questionnaires } \\
\text { Depression, PHQ-9 }\end{array}$ & $6.45 \pm 6.05$ & $1.00 \pm 1.80$ & $<0.00 I^{*}$ \\
$\begin{array}{l}\text { Anxiety, HADS } \\
\text { Family support, APGAR } \\
\text { index }\end{array}$ & $2.27 \pm 2.88$ & $1.94 \pm 2.63$ & 0.714 \\
PTSD, SPAN-C & $2.36 \pm 2.34$ & $7.53 \pm 2.40$ & 0.282 \\
Health condition, EQ-5D & $59.55 \pm 1.96$ & $0.29 \pm 0.69$ & 0.116 \\
VAS & 8.45 & $75.29 \pm 14.19$ & $0.008^{*}$ \\
Sleep, PSQI Global score & $8.31 \pm 5.88$ & $4.43 \pm 2.53$ & $0.002^{*}$ \\
Fatigue, BFI-T & $3.76 \pm 4.80$ & $1.69 \pm 3.67$ & 0.149 \\
\hline Salivary stress & & & \\
hormones & & & \\
DHEA (Pg/mL) & $75.00 \pm 63.31$ & $167.56 \pm 127.16$ & $0.012^{*}$ \\
DHEAS (ng/mL) & $2.12 \pm 1.32$ & $3.29 \pm 1.96$ & $0.032^{*}$ \\
Cortisol (ug/d) & $0.14 \pm 0.10$ & $0.18 \pm 0.09$ & 0.637 \\
\hline
\end{tabular}

Note: $* P<0.05$.

Abbreviations: PHQ-9, Patient Health Questionnaire; HADS, Hospital Anxiety and Depression Scale; PTSD, post-traumatic stress disorder; SPAN-C, Startle, Physiological arousal, Anger, and Numbness-Chinese version; EQ-5D VAS, EQ-5D visual analog scale; PSQI, Pittsburgh Sleep Quality Index; BFI-T, Brief Fatigue Inventory, Taiwan version; DHEA, dehydroepiandrosterone; DHEAS, dehydroepiandrosterone sulfate.

and depression scores were significantly negatively correlated with the salivary concentrations of DHEAS $(r=$ $-0.496, P=0.019 ; r=-0.562, P=0.006$, respectively, Table 3 ). The post-CT group exhibited lower salivary concentrations of DHEAS and higher fatigue and depression scores than did the control group (Table 3, Figure 1).

\section{Discussion}

The present study showed that the salivary concentrations of DHEA and DHEAS decreased in patients with NSCLC after CT. A low salivary DHEAS concentration was associated with more fatigue and depression among patients with NSCLC after CT.

In healthy individuals, DHEAS concentrations peak during youth and subsequently decrease with age. Patients with metastatic cancers have lower concentrations of serum DHEAS than patients without metastatic cancers or healthy individuals; ${ }^{14}$ furthermore, patients with advanced stages of cancer exhibit a greater decline in endogenous DHEAS production than did those with the early stages of cancer. ${ }^{15}$ The median age of lung cancer diagnosis is 70 years in men and women, and $37 \%$ of patients with lung cancer are older than 75 years.
Table 3 Correlation Between Hormone Concentrations and Mental Health Among Patients with Lung Cancer After Chemotherapy

\begin{tabular}{|l|l|l|l|l|}
\hline & & DHEA & DHEAS & Cortisol \\
\hline Depression, PHQ-9 & $r$ & $\begin{array}{l}-0.204 \\
0.363\end{array}$ & $\begin{array}{l}-0.496^{*} \\
0.019^{*}\end{array}$ & $\begin{array}{l}-0.125 \\
0.609\end{array}$ \\
\hline Anxiety, HADS & $P$ & 0.028 & -0.043 & -0.030 \\
& $P$ & 0.902 & 0.848 & 0.903 \\
\hline Family support, APGAR & $r$ & 0.101 & -0.012 & 0.246 \\
index & $P$ & 0.654 & 0.958 & 0.310 \\
\hline PTSD, SPAN-C & $R$ & -0.107 & -0.202 & -0.037 \\
& $P$ & 0.637 & 0.367 & 0.882 \\
\hline Health condition, EQ-5D & $r$ & 0.070 & 0.193 & 0.154 \\
VAS & $P$ & 0.757 & 0.390 & 0.530 \\
\hline Sleep, PSQI global score & $r$ & -0.350 & -0.350 & -0.377 \\
& $P$ & 0.184 & 0.184 & 0.204 \\
\hline Fatigue, BFI-T & $r$ & -0.298 & $-0.562^{*}$ & 0.207 \\
& $P$ & 0.178 & $0.006^{*}$ & 0.394 \\
\hline
\end{tabular}

Note: $* P<0.05$.

Abbreviations: PHQ-9, Patient Health Questionnaire-9; HADS, Hospital Anxiety and Depression Scale; PTSD, post-traumatic stress disorder; SPAN-C, Startle, Physiological arousal, Anger, and Numbness-Chinese version; EQ-5D VAS, EQ-5D visual analog scale; PSQI, Pittsburgh Sleep Quality Index; BFI-T, Brief Fatigue Inventory, Taiwan version.

Furthermore, most patients with lung cancer are at an advanced stage of the disease (stage III/IV) at diagnosis. Thus, patients with lung cancer are expected to have lower DHEA and DHEAS concentrations because of old age and advanced disease status. In a previous study, DHEA and DHEAS concentrations were significantly lower during targeted therapy with gefitinib than pretreatment values in patients with lung cancer. ${ }^{16}$ However, few reports on patients with lung cancer receiving CT are currently available. In the present study, post-CT patients had significantly lower DHEA and DHEAS concentrations than did healthy controls.

DHEA and DHEAS are androgen precursors synthesized and secreted primarily by the adrenal cortex and are involved in the HPA axis. Because DHEAS has a longer half-life and fewer circadian variations than DHEA, ${ }^{28}$ its concentration can be used to evaluate the integrity of the HPA axis. ${ }^{13,29}$ Low concentrations of DHEA and DHEAS are associated with metabolic and cardiovascular diseases, decline in muscle mass, poor physical performance, mood changes, and memory deficits. ${ }^{13}$ In a recent study, Strasser et al enrolled 48 patients with advanced cancer who did not receive major anticancer intervention. Low concentrations of free testosterone 
A

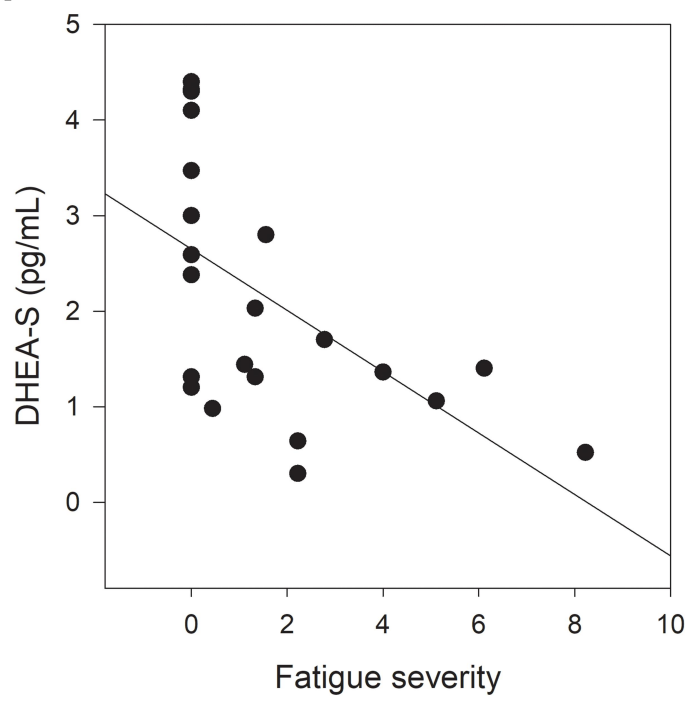

B

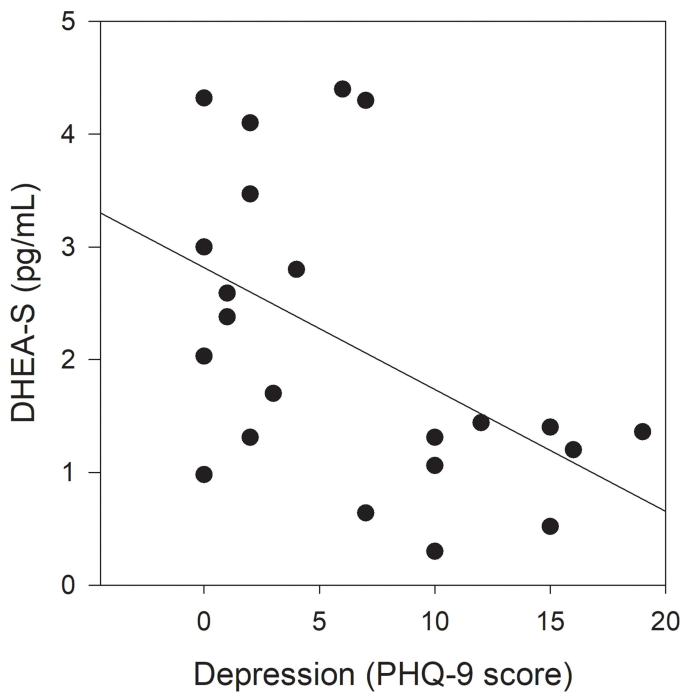

Figure I The scatter plots and regression line of salivary DHEAS concentration versus PHQ-9 scores and fatigue severity of post-CT lung cancer patients.

(hypogonadism) were correlated with more fatigue, anxiety, depression, and low emotional well-being scores. The study demonstrated that hypogonadism contributed to fatigue and negative mood symptoms. ${ }^{30}$ In the present study, patients in the post-CT group with lower salivary DHEAS concentrations exhibited more depression and fatigue. Our findings were essentially comparable with those of previous studies.

Depression is highly associated with the diagnosis of cancer, but currently, the biology of depression in cancer patients was still controversial. Three of the prominent theories including inflammatory mediators, overactive HPA axis and excess concentrations of the neurotransmitter glutamate. ${ }^{11}$ In a case-control and meta-analysis study, decreased serum DHEAS levels are associated with the development of depression and it decreased with the rising of age. ${ }^{31}$ In a randomized control trial, using 6 weeks of DHEA demonstrated efficacy for midlife-onset major and minor depression. ${ }^{32}$ In addition, untreated Addison's patients had subnormal DHEAS levels, and DHEA replacement was reported to improve mood and fatigue scores among patients with Addison's disease in a double-blind randomized trial. ${ }^{33}$ DHEA may be one of the effective alternative drugs used in the treatment of depression. ${ }^{34}$

Cancer-related fatigue (CRF) is the subjective sense of tiredness or exhaustion related to cancer or cancer treatment. ${ }^{35}$ Fatigue commonly occurs with symptoms such as pain, depression, and sleep disturbance and results in the impairment of physical activity, functional status, and quality of life (QoL). Increased fatigue is also associated with low survival rates among patients with lung cancer. ${ }^{36}$ Current theories regarding mechanisms underlying CRF include hypothalamic-pituitary-adrenal (HPA) axis dysfunction, neurotransmitter or cytokine dysregulation, alterations in muscle and adenosine triphosphate metabolism, and circadian rhythm alternations. The neuroendocrine system, which includes the autonomic nervous system and HPA axis, plays a major role in managing immune or inflammatory processes and is associated with chronic fatigue syndrome in addition to CRF. ${ }^{10}$

Gender would be an important factor to influence DHEA and DHEAS but the results of previous studies were inconclusive. One study enrolled 338 African American and Caucasian women, and reported lower DHEAS levels were negatively associated with depressive symptoms in the women in the older half of this cohort but the positive relationship in younger women. ${ }^{37}$ Another study reported DHEAS levels were significantly and inversely associated with depressed mood in older women. ${ }^{38}$ Among patients with major depression disorder received medical therapy; serum levels of DHEAS in male patients were significantly decreased compared with male controls, whereas no significant differences were seen in female patients and controls. ${ }^{39} 70-75 \%$ of female lung cancer patients in Taiwan had druggable EGFR/ALK/ROS1 mutant and received targeted therapy as first-line therapy. In our present study, patients under targeted therapy were not enrolled. Thus, there were 16 male but only 6 female. The concentrations of hormones had no statistically significant difference in both genders in our present study, but this might be because of small numbers of participants. 
The present study has several limitations. First, this study was limited by its cross-sectional design and relatively small sample size. We did not have access to longitudinal data in this pilot study. Second, apart from CT, numerous other factors may cause physiological and psychological stress and result in changes in the salivary concentrations of stress hormone. We cannot conclude that CT causes low DHEA and DHEAS concentrations based on this cross-sectional analysis. Third, patients with extreme fatigue frequently experience severe discomfort simultaneously. Patients usually do not agree to sign informed consent forms and encounter difficulties in completing questionnaires. The lack of data from patients experiencing severe fatigue may have caused the underestimation of the relationship between hormone concentrations and fatigue.

\section{Conclusions}

The salivary concentrations of DHEA and DHEAS were lower in patients with NSCLC after CT than in healthy controls. Lower salivary DHEAS concentrations were associated with higher fatigue and depression scores among patients with lung cancer after CT.

\section{Abbreviations}

CRF, cancer-related fatigue; CT, chemotherapy; QoL, quality of life; HPA axis, hypothalamic-pituitary-adrenal axis; DHEA, dehydroepiandrosterone; DHEAS, DHEA sulfate; NSCLC, non-small-cell lung cancer; ECOG, Eastern Cooperative Oncology Group; IRB, institutional review board.

\section{Acknowledgments}

This study was supported by grants from Chang Gung Memorial Hospital, Chiayi, Taiwan (grant number: CORPG6G0101, CORPG6G0151). The funder had no role in the design and conduct of the study; collection, management, analysis, and interpretation of data; preparation, review, or approval of the manuscript; and the decision to submit the manuscript for publication.

\section{Disclosure}

The authors report no conflicts of interest in this work.

\section{References}

1. Barta JA, Powell CA, Wisnivesky JP. Global epidemiology of lung cancer. Ann Glob Health. 2019;85(1).

2. Takano N, Ariyasu R, Koyama J, et al. Improvement in the survival of patients with stage IV non-small-cell lung cancer: experience in a single institutional 1995-2017. Lung Cancer. 2019;131:69-77. doi:10.1016/j.lungcan.2019.03.008
3. Vodermaier A, Lucas S, Linden W, et al. Anxiety after diagnosis predicts lung cancer-specific and overall survival in patients with stage III non-small cell lung cancer: a population-based cohort study. $J$ Pain Symptom Manage. 2017;53(6):1057-1065. doi:10.1016/j.jpainsymman.2016.12.338

4. Doroshow DB, Herbst RS. Treatment of advanced non-small cell lung cancer in 2018. JAMA Oncol. 2018;4(4):569-570. doi:10.1001/ jamaoncol.2017.5190

5. Stone P, Richards M, A'Hern R, et al. A study to investigate the prevalence, severity and correlates of fatigue among patients with cancer in comparison with a control group of volunteers without cancer. Ann Oncol. 2000;11(5):561-568. doi:10.1023/A:10083 31230608

6. Zabora J, BrintzenhofeSzoc K, Curbow B, et al. The prevalence of psychological distress by cancer site. Psychooncology. 2001;10 (1):19-28. doi:10.1002/1099-1611(200101/02)10:1<19::AIDPON501>3.0.CO;2-6

7. Park B, Youn S, Yi -K-K, et al. The prevalence of depression among patients with the top ten most common cancers in South Korea. Psychiatry Investig. 2017;14(5):618-625. doi:10.4306/pi.2017.1 4.5.618

8. Pearce A, Haas M, Viney $R$, et al. Incidence and severity of self-reported chemotherapy side effects in routine care: a prospective cohort study. PLoS One. 2017;12(10):e0184360. doi: 10.1371 /journal.pone. 0184360

9. Walling AM, Weeks JC, Kahn KL, et al. Symptom prevalence in lung and colorectal cancer patients. J Pain Symptom Manage. 2015;49 (2):192-202. doi:10.1016/j.jpainsymman.2014.06.003

10. Bower JE. The role of neuro-immune interactions in cancer-related fatigue: biobehavioral risk factors and mechanisms. Cancer. 2019;125(3):353-364. doi:10.1002/cncr.31790

11. Young K, Singh G. Biological mechanisms of cancer-induced depression. Front Psychiatry. 2018;9:299. doi:10.3389/fpsyt.201 8.00299

12. Rutkowski K, Sowa P, Rutkowska-Talipska J, et al. Dehydroepiandrosterone (DHEA): hypes and hopes. Drugs. 2014;74 (11):1195-1207. doi:10.1007/s40265-014-0259-8

13. Fischli S, Jenni S, Allemann S, et al. Dehydroepiandrosterone sulfate in the assessment of the hypothalamic-pituitary-adrenal axis. J Clin Endocrinol Metab. 2008;93(2):539-542. doi:10.1210/jc.2007-1780

14. Lissoni P, Rovelli F, Giani L, et al. Dehydroepiandrosterone sulfate (DHEAS) secretion in early and advanced solid neoplasms: selective deficiency in metastatic disease. Int J Biol Markers. 1998;13 (3):154-157. doi:10.1177/172460089801300306

15. Bhatavdekar JM, Patel DD, Chikhlikar PR, et al. Levels of circulating peptide and steroid hormones in men with lung cancer. Neoplasma. 1994;41(2):101-103.

16. Nishio M, Ohyanagi F, Horiike A, et al. Gefitinib treatment affects androgen levels in non-small-cell lung cancer patients. Br J Cancer. 2005;92(10):1877-1880. doi:10.1038/sj.bjc.6602585

17. Ettinger DS, Wood DE, Aggarwal C, et al. NCCN guidelines insights: non-small cell lung cancer, version 1.2020. J Natl Compr Canc Netw. 2019;17(12):1464-1472. doi:10.6004/jnccn.2019.0059

18. Edge SB, Compton CC. The American Joint Committee on cancer: the 7th edition of the AJCC cancer staging manual and the future of TNM. Ann Surg Oncol. 2010;17(6):1471-1474. doi:10.1245/s10434010-0985-4

19. de Kock I, Mirhosseini M, Lau F, et al. Conversion of Karnofsky Performance Status (KPS) and Eastern Cooperative Oncology Group Performance Status (ECOG) to Palliative Performance Scale (PPS), and the interchangeability of PPS and KPS in prognostic tools. $J$ Palliat Care. 2013;29(3):163-169. doi:10.1177/082585971 302900305

20. Kroenke K, Spitzer RL, Williams JB. The PHQ-9: validity of a brief depression severity measure. J Gen Intern Med. 2001;16(9):606-613. doi:10.1046/j.1525-1497.2001.016009606.x 
21. Bellon Saameno JA, Delgado Sánchez A, Luna Del Castillo JD, et al. [Validity and reliability of the family Apgar family function test] Aten Primaria. 1996;18(6):289-296.[Article in Spanish]

22. Meltzer-Brody S, Churchill E, Davidson JR. Derivation of the SPAN, a brief diagnostic screening test for post-traumatic stress disorder. Psychiatry Res. 1999;88(1):63-70. doi:10.1016/S0165-1781(99) 00070-0

23. Chen $\mathrm{CH}$, Shen WW, Tan HK-L, et al. The validation study and application of stratum-specific likelihood ratios in the Chinese version of SPAN. Compr Psychiatry. 2003;44(1):78-81. doi:10.1053/ comp.2003.50013

24. Chang TJ, Tarn Y-H, Hsieh C-L, et al. Taiwanese version of the EQ-5D: validation in a representative sample of the Taiwanese population. J Formos Med Assoc. 2007;106(12):1023-1031. doi:10.1016/S0929-6646(08)60078-9

25. Lin CC, Chang A-P, Chen M-L, et al. Validation of the Taiwanese version of the brief fatigue inventory. J Pain Symptom Manage. 2006;32(1):52-59. doi:10.1016/j.jpainsymman.2005.12.019

26. Tzeng JI, Fu YW, Lin CC. Validity and reliability of the Taiwanese version of the Pittsburgh sleep quality index in cancer patients. Int $J$ Nurs Stud. 2012;49(1):102-108. doi:10.1016/j.ijnurstu.20 11.08 .004

27. Lee Y, Wu Y-S, Chien C-Y, et al. Use of the hospital anxiety and depression scale and the Taiwanese depression questionnaire for screening depression in head and neck cancer patients in Taiwan. Neuropsychiatr Dis Treat. 2016;12:2649-2657. doi:10.2147/NDT. S112069

28. van Leeuwen RW, Peric R, Hussaarts KGAM, et al. Influence of the acidic beverage cola on the absorption of erlotinib in patients with non-small-cell lung cancer. J Clin Oncol. 2016;34(12):1309-1314. doi: 10.1200/JCO.2015.65.2560

29. Charoensri S, Chailurkit L, Muntham D, et al. Serum dehydroepiandrosterone sulfate in assessing the integrity of the hypothalamic-pituitary-adrenal axis. $J$ Clin Transl Endocrinol. 2017;7:42-46. doi:10.1016/j.jcte.2017.01.001

30. Strasser F, Palmer JL, Schover LR, et al. The impact of hypogonadism and autonomic dysfunction on fatigue, emotional function, and sexual desire in male patients with advanced cancer: a pilot study. Cancer. 2006;107(12):2949-2957. doi:10.1002/cncr.22339
31. Souza-Teodoro LH, de Oliveira C, Walters K, et al. Higher serum dehydroepiandrosterone sulfate protects against the onset of depression in the elderly: findings from the English Longitudinal Study of Aging (ELSA). Psychoneuroendocrinology. 2016;64:40-46. doi:10.1016/j.psyneuen.2015.11.005

32. Schmidt PJ, Daly RC, Bloch $\mathrm{M}$, et al. Dehydroepiandrosterone monotherapy in midlife-onset major and minor depression. Arch Gen Psychiatry. 2005;62(2):154-162. doi:10.1001/archpsyc.62.2.154

33. Hunt PJ, Gurnell EM, Huppert FA, et al. Improvement in mood and fatigue after dehydroepiandrosterone replacement in Addison's disease in a randomized, double blind trial. J Clin Endocrinol Metab. 2000;85(12):4650-4656. doi:10.1210/jcem.85.12.7022

34. Peixoto C, Devicari Cheda J, Nardi A, et al. The effects of dehydroepiandrosterone (DHEA) in the treatment of depression and depressive symptoms in other psychiatric and medical illnesses: a systematic review. Curr Drug Targets. 2014;15(9):901-914. doi:10.2174/1389450115666140717111116

35. Berger AM, Mooney K, Alvarez-Perez A, et al. Cancer-related fatigue, version 2.2015. J Natl Compr Canc Netw. 2015;13 (8):1012-1039. doi:10.6004/jnccn.2015.0122

36. Scott HR, McMillan DC, Forrest LM, et al. The systemic inflammatory response, weight loss, performance status and survival in patients with inoperable non-small cell lung cancer. $\mathrm{Br} J$ Cancer. 2002;87(3):264-267. doi:10.1038/sj.bjc.6600466

37. Morrison MF, Ten Have T, Freeman EW, et al. DHEA-S levels and depressive symptoms in a cohort of African American and Caucasian women in the late reproductive years. Biol Psychiatry. 2001;50 (9):705-711. doi:10.1016/S0006-3223(01)01169-6

38. Barrett-Connor E, von Mühlen D, Laughlin GA, et al. Endogenous levels of dehydroepiandrosterone sulfate, but not other sex hormones, are associated with depressed mood in older women: the Rancho Bernardo Study. J Am Geriatr Soc. 1999;47(6):685-691. doi:10.1111/j.1532-5415.1999.tb01590.x

39. Kurita H, Maeshima H, Kida S, et al. Serum dehydroepiandrosterone (DHEA) and DHEA-sulfate (S) levels in medicated patients with major depressive disorder compared with controls. J Affect Disord. 2013;146(2):205-212. doi:10.1016/j.jad.2012.09.004
Neuropsychiatric Disease and Treatment

\section{Publish your work in this journal}

Neuropsychiatric Disease and Treatment is an international, peerreviewed journal of clinical therapeutics and pharmacology focusing on concise rapid reporting of clinical or pre-clinical studies on a range of neuropsychiatric and neurological disorders. This journal is indexed on PubMed Central, the 'PsycINFO' database and CAS, and is the official journal of The International Neuropsychiatric Association (INA). The manuscript management system is completely online and includes a very quick and fair peer-review system, which is all easy to use. Visit http://www.dovepress.com/testimonials.php to read real quotes from published authors. 